\title{
Ab initio properties of Li-Group-II molecules for ultracold matter studies
}

\author{
Svetlana Kotochigova * Alexander Petrov ${ }^{\dagger}$ and Maria Linnik \\ Department of Physics, Temple University, Philadelphia, PA 19122, USA \\ Jacek Kłos \\ Department of Chemistry and Biochemistry, University of Maryland, College Park, MD 20742, USA \\ Paul S. Julienne \\ Joint Quantum Institute, NIST and University of Maryland, Gaithersburg, MD 20899-8423, USA
}

\begin{abstract}
We perform a systematic investigation of the electronic properties of the ${ }^{2} \Sigma^{+}$ground state of Lialkaline-earth dimers. These molecules are proposed as possible candidates for quantum simulation of lattice-spin models. We apply powerful quantum chemistry coupled-cluster method and large basis sets to calculate potential energies and permanent dipole moments for the $\mathrm{LiBe}, \mathrm{LiMg}, \mathrm{LiCa}$, $\mathrm{LiSr}$, and LiYb molecules. Agreement of calculated molecular constants with existing experimental data is better than or equal to $8 \%$. Our results reveal a surprising irregularity in the dissociation energy and bond length with an increase in the reduced mass of the molecule. At the same time the permanent dipole moment at the equilibrium separation has the smallest value between 0.01 a.u. and 0.1 a.u. for the heaviest ( $\mathrm{LiSr}$ and $\mathrm{LiYb}$ ) molecules and increases to 1.4 a.u. for the lightest $(\mathrm{LiBe})$, where $1 \mathrm{a}$.u. is one atomic unit of dipole moment. We consider our study of the ${ }^{2} \Sigma^{+}$molecules a first step towards a comprehensive analysis of their interactions in an optical trap.
\end{abstract}

\section{INTRODUCTION}

There is growing interest in molecules formed by one alkali-metal atom and one alkaline-earth or rare-earth atom [1]11. In contrast to group I or group II diatomic molecules, these molecules have an unpaired electron that allows for manipulation of the $\mathrm{X}^{2} \Sigma^{+}$groundstate molecule with both electric and magnetic external fields. Moreover, it has been suggested that ${ }^{2} \Sigma^{+}$ molecules are good candidates in which to explore controlled chemical reactions at ultracold temperatures [12]. The unpaired spin in the ground state of these molecules should provide a new handle for the control of the reaction dynamics based on the interplay between intramolecular spin-rotation couplings. In addition, when placed in an optical lattice, these polar molecules can interact with each other via both electric dipole-dipole and magnetic spin-spin forces. It is therefore possible to engineer unusual forms of interactions for quantum simulation of lattice-spin models and topological quantum computing 1], controlled preparation of many-body entangled states [4, and high-precision measurements of fundamental constants [13].

Another interest in Li-group II molecules stems from prospects to achieve optical Feshbach tuning of scattering properties in ultracold gases of the individual atoms with-

\footnotetext{
*Corresponding author: skotoch@temple.edu

${ }^{\dagger}$ Alternative address: St. Petersburg Nuclear Physics Institute, Gatchina, 188300; Department of Physics, St.Petersburg State University, 198904, Russia
}

out substantial loss of atoms from a trap. This photoassociative tuning, pioneered for homonuclear molecules [1421, becomes possible due to the existence of long-lived excited molecular states near narrow intercombination lines of the alkaline-earth or rare-earth atoms. In turn, this might enable efficient ways to form gases of polar molecules without substantial loss by photoassociation. In essence, a two-photon optical Feshbach resonance can be used to couple two colliding atoms to a vibrational level of the molecular ground state. The suppressed effect of excited-state spontaneous decay makes efficient coherent molecular formation possible. Knowledge of the electronic and ro-vibrational properties of the molecules will help to find optimal pathways. Characteristics such as the permanent dipole moments of the ground state will determine the anisotropic interactions between these molecules in an optical trap.

Some key theoretical predictions of the electronic structure and dipole moment of the ground and low-lying excited states of LiYb [8, 9] and for the ground state of LiSr 6] have recently been obtained. Other work has studied RbYb [2] and RbSr [5]. However, the physical origin of the chemical bond of these mixed species is not yet fully understood. Continued theoretical advances are necessary to unravel the properties of these molecular systems. To the best of our knowledge, only the $\mathrm{LiMg}$ and $\mathrm{LiCa}$ ground states were experimentally investigated [11, 22, 23, which allows us to compare our predictions with experimentally obtained molecular parameters [11, 23. There are several experimental groups working to achieve the formation of the $\mathrm{LiYb}$ molecules at ultracold temperatures [7, 10]. An understanding and quantitative description of these molecules might help to 
define conditions under which these ultracold molecular systems can be created.

Our theoretical study is devoted to a systematic investigation of the $\mathrm{X}^{2} \Sigma^{+}$ground state properties of the polar Li-alkaline-earth dimers such as LiBe, LiMg, LiCa, LiSr, and the rare-earth dimer LiYb. These are interesting and challenging systems as the valence electrons of the atoms form a relatively weak covalent bond [25]. The alkaline-earth atom has a closed outer electron shell and a Hartree-Fock interaction energy results in a purely repulsive potential curve. We use the unrestricted (U) or partially spin-restricted (R) coupled cluster (CC) method with either single and double excitations augmented with pertubatively calculated triples $(\mathrm{CCSD}(\mathrm{T}))$ or with single, double, and triple excitations (CCSDT) to treat the correlations of core and valence electrons. All approaches are used in conjunction with correlation-consistent basis sets. The dipole moment is calculated using a finite field approach with a four-point numerical differentiation formula. The calculations are performed with CFOUR [26] and MOLPRO [27] suites of programs.

Presumably, the CCSDT method is more accurate in the description of the potential energy surface than the CCSD(T) method, where triple excitations are treated perturbatively. The results of the unrestricted (U) method are more accurate than that of a restricted $(\mathrm{R})$ calculation. The $\mathrm{CC}$ method is based on a singlereference configuration and is not suitable for molecules where multiple-configurations are relevant. A practical indicator of how well the CC method performs is based on $\mathrm{T} 1$ diagnostics [28. Our calculation shows that T1 is small and varies from 0.015 to 0.035 . This indicates that a single-reference method is valid for these dimers. In fact, a small T1 for all interatomic separations implies that the long-range interaction potentials as well as the dissociation energies can be accurately described.

Our results include the ground-state potential curve, bond distance, harmonic frequency, dissociation energy, and permanent dipole moment for $\mathrm{LiBe}, \mathrm{LiMg}, \mathrm{LiCa}$, LiSr, and LiYb. For completeness, we calculate vibrational energies of the ground-state potentials. Due to limitations in computational resources we were not able to obtain results for all methods, basis sets, and Li-X diatoms. The most accurate potential energy curves and dipole moments are presented in the figures and tables with the exception of Tab.VI, which compares molecular constants obtained with the various methods and basis sets.

All potentials are corrected for basis set superposition errors (BSSE) using the counterpoise procedure of Boys and Bernardi 33 . The sign of the dipole moment depends on the position of the origin. We apply the following convention throughout the paper: The alkalineearth/rare-earth atom is positioned at the origin and the Li atom moves along the positive $x$-axis. A positive sign of the dipole moment corresponds to the charge transfer from the $\mathrm{Li}$ atom towards the alkaline-earth/rare-earth atom.

\section{A. LiBe molecule}

There exist several theoretical calculations of the $\mathrm{X}^{2} \Sigma_{1 / 2}^{+}$electronic ground state of the LiBe molecule. Jones [29] used a density functional formalism based on a local density approximation to predict molecular constants of the LiBe, LiMg, and LiCa molecules. Their dissociation energy is $D_{e} /(h c)=3705.9 \mathrm{~cm}^{-1}$, while the equilibrium bond length $R_{e}$ is $4.9 a_{0}$ and the vibrational constant $\omega_{e} /(h c)=340 \mathrm{~cm}^{-1}$. Such strong bonding of the LiBe molecule was explained by substantial electroncharge transfer between s and p orbitals. More advanced MRCI calculations of the low-lying doublet and quartet states of LiBe was performed by Fischer et al. [30]. In this calculation the ground state is bound by 2497.5 $\mathrm{cm}^{-1}, R_{e}=4.95 a_{0}$, and $\omega_{e} /(h c)=302 \mathrm{~cm}^{-1}$. The permanent dipole moment is $d_{e}=0.95$ a.u. at $R_{e}$. Another MRCI calculation 25] using all single and double excitations obtained $D_{e} /(h c)=2336.35 \mathrm{~cm}^{-1}, R_{e}=4.927 a_{0}$, and $\omega_{e} /(h c)=300 \mathrm{~cm}^{-1}$, which are close to those of Ref. 30]. However, the dipole moment $d_{e}=1.33$ a.u. of Ref. 25] differs significantly from that of Ref. [30]. The effect of inner-shell correlations on the molecular electronic structure was investigated. Later an ab initio configuration-interaction study was reported by Marino and Ermler 31. Their value of the dissociation energy $D_{e} /(h c)=2014 \mathrm{~cm}^{-1}$ is smaller than that in previous studies. In summary, the previous theoretical calculations disagree on dissociation energy and dipole moment values but have much better agreement on the value of the bond length. Our goal is to test results of the previous predictions by performing a coupled cluster calculation accounting for high-order correlation effects.

In our calculation the $\operatorname{UCCSD}(\mathrm{T})$ method is used to obtain the ground state potential and dipole moment of LiBe as a function of interatomic separation $R$. These characteristics are calculated with the large basis set (aug-cc-pV5Z-DK) of Prascher et al [35], which includes scalar relativistic effects accounted for by the Douglass-Kroll Hamiltonian. Atomic basis sets include (15s8p5d4f3g2h) primitive Gaussian and $[7 \mathrm{~s} 6 \mathrm{p} 5 \mathrm{~d} 4 \mathrm{f} 3 \mathrm{~g} 2 \mathrm{~h}]$ constructed functions for $\mathrm{Li}$ and (15s9p5d4f3g2h)/[7s6p5d4f3g2h] for Be.

Results of our calculation are shown in Figure 1. Comparing our data with results available from other theories and experiment we conclude that $R_{e}=4.87 a_{0}$ is close to the experimental measurement of $R_{e}=4.894 a_{0}$ 32, while the well depth $D_{e} /(h c)=2406.89 \mathrm{~cm}^{-1}$ is in best agreement with the prediction of [25]. In Ref. 32] no value of $D_{e}$ is given. The dipole moment value $d_{e}$ $=1.41$ a.u. at the equilibrium separation is even larger than that of 25]. The permanent dipole moment averaged over the ground state vibrational wave function is equal to 1.37 a.u. It is slightly lower than the value of the dipole moment function at $R=R_{e}$. The LiBe molecule has the largest dipole moment of the molecular systems considered in this work.

Bound states of ${ }^{7} \mathrm{Li}^{9} \mathrm{Be}$ are calculated using a discrete 


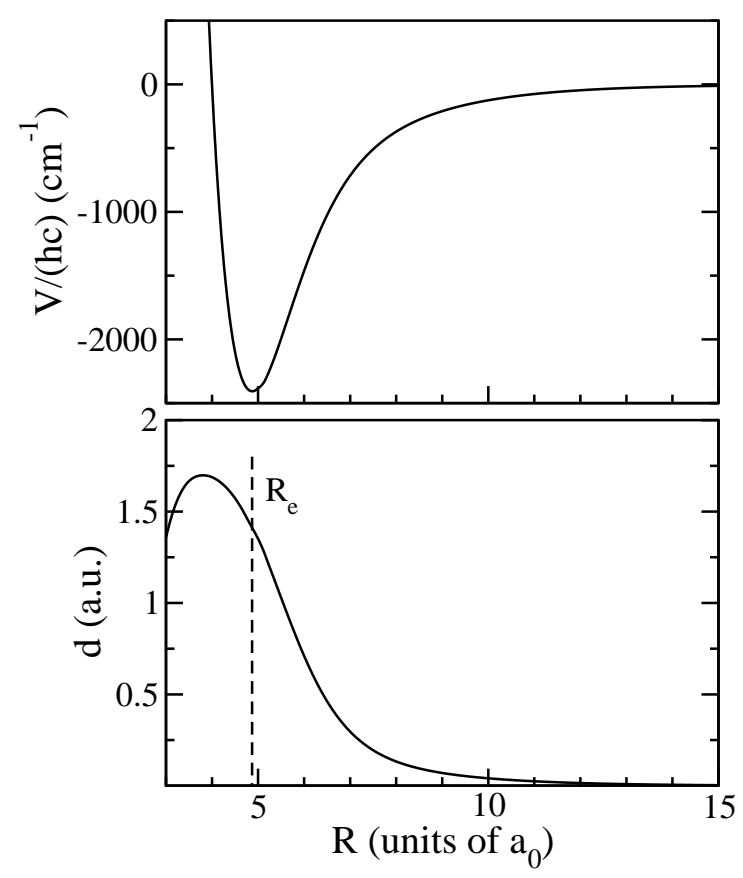

FIG. 1: Top panel: electronic ${ }^{2} \Sigma^{+}$ground-state potential of LiBe; bottom panel: permanent dipole moment function of the ground state of $\mathrm{LiBe}$. The vertical dashed line indicates the equilibrium separation of the ground state potential, at which the dipole moment equals 1.41 a.u. The vibrationallyaveraged dipole moment of the $v=0$ level is equal to a slightly smaller value of 1.372 a.u.

TABLE I: All vibrational energies of the ${ }^{7} \mathrm{Li}^{9} \mathrm{Be} \mathrm{X}^{2} \Sigma^{+}$ground state potential for the rotational state $N=0$.

\begin{tabular}{cccc}
\hline \hline$\nu$ & Energy/(hc) $\left(\mathrm{cm}^{-1}\right)$ & $\nu$ & Energy/(hc) $\left(\mathrm{cm}^{-1}\right)$ \\
0 & -2254.29 & 9 & -337.24 \\
1 & -1954.76 & 10 & -243.42 \\
2 & -1669.25 & 11 & -169.45 \\
3 & -1407.21 & 12 & -112.19 \\
4 & -1167.64 & 13 & -68.88 \\
5 & -951.37 & 14 & -37.48 \\
6 & -760.21 & 15 & -16.55 \\
7 & -594.38 & 16 & -4.86 \\
8 & -453.64 & 17 & -0.50 \\
\hline \hline
\end{tabular}

variable representation (DVR) 36, and their binding energies are presented in Table I. The zero-point energy is found to be $D_{0} /(h c)=2254.29 \mathrm{~cm}^{-1}$.

\section{B. LiMg molecule}

To the best of our knowledge, there exist two experimental investigations of the LiMg ground state proper- ties [22, 23]. The study in Ref. 23] provides molecular constants that we can use for comparison with our calculation. This work was based on resonant photo-ionization spectroscopy of LiMg in the gas phase and gives an accurate bond energy $D_{0} /(h c)=1330 \mathrm{~cm}^{-1}$, equilibrium separation $R_{e}=5.9 a_{0}$, and vibrational frequency $\omega_{e} /(h c)$ $=190 \mathrm{~cm}^{-1}$. A density functional study in Ref. [29] of LiMg gives $D_{e} /(h c)=1371 \mathrm{~cm}^{-1}, R_{e}=6.04 a_{0}$, and $\omega_{e} /(h c)=180 \mathrm{~cm}^{-1}$. A MRCI approach with large contracted basis sets and multiple sets of polarization functions is used in Ref. 25] to give predictions for the $\mathrm{LiMg}$ ground state molecular constants $D_{e} /(h c)=1611.28$ $\mathrm{cm}^{-1}, R_{e}=5.88 a_{0}$, and $\omega_{e} /(h c)=183 \mathrm{~cm}^{-1}$. The value for $D_{e}$ significantly differs from both Ref. [29] and experiment.

Here we apply RCCSD(T) and $\operatorname{UCCSD}(\mathrm{T})$ methods with two different basis sets to calculate the potential and dipole moment of the $\mathrm{LiMg}$ ground state. Augmented polarized core-valence (aug-ccpCVQZ) basis sets with Li:(15s9p5d3f1g)/[8s7p5d3f1g] and Mg:(19s15p6d4f2g)/[9s8p6d4f2g] functions [34, 35] are used to calculate the ground state potential energy at the RCCSD(T) level of theory, where all electrons in both $\mathrm{Li}$ and $\mathrm{Mg}$ are correlated. Aug-cc-pv5z-DK basis sets with Li: (14s8p4d3f2g1h)/[6s5p4d3f2g1h] and Mg: (20s14p4d3f2g1h)/[7s6p4d3f2g1h] [35 are applied in the $\mathrm{UCCSD}(\mathrm{T})$ calculation. The calculation is optimized for scalar relativistic calculations of the all-electron DouglasKroll Hamiltonian. A comparison with the molecular constants measured in Ref. [23] shows that our $D_{0}$ and $R_{e}$ values are in good agreement with this experiment. Figure 2 shows the $R$-dependent potential and dipole moment.

In Table II we present a complete list of $N=0$ bound states of the $\mathrm{X}^{2} \Sigma^{+}$ground state potential of ${ }^{7} \mathrm{Li}^{24} \mathrm{Mg}$ obtained by a DVR calculation. The zero-point corrected energy is found to be $D_{0} /(h c)=1330.05 \mathrm{~cm}^{-1}$ in agreement with experimental measurement [23].

TABLE II: All vibrational energies of the ${ }^{7} \mathrm{Li}^{24} \mathrm{Mg} \mathrm{X}^{2} \Sigma^{+}$ ground state potential for the $N=0$ rotational state. The potential is based on the $\operatorname{RCCSD}(\mathrm{T})$ calculation.

\begin{tabular}{cccc}
\hline \hline$\nu$ & Energy/(hc) $\left(\mathrm{cm}^{-1}\right)$ & $\nu$ & Energy $/(h c)\left(\mathrm{cm}^{-1}\right)$ \\
0 & -1330.05 & 10 & -216.21 \\
1 & -1164.35 & 11 & -164.06 \\
2 & -1010.96 & 12 & -120.16 \\
3 & -870.00 & 13 & -83.91 \\
4 & -741.52 & 14 & -54.87 \\
5 & -625.41 & 15 & -32.62 \\
6 & -521.43 & 16 & -16.78 \\
7 & -429.16 & 17 & -6.89 \\
8 & -348.00 & 18 & -1.75 \\
9 & -277.27 & 19 & -0.12 \\
\hline \hline
\end{tabular}




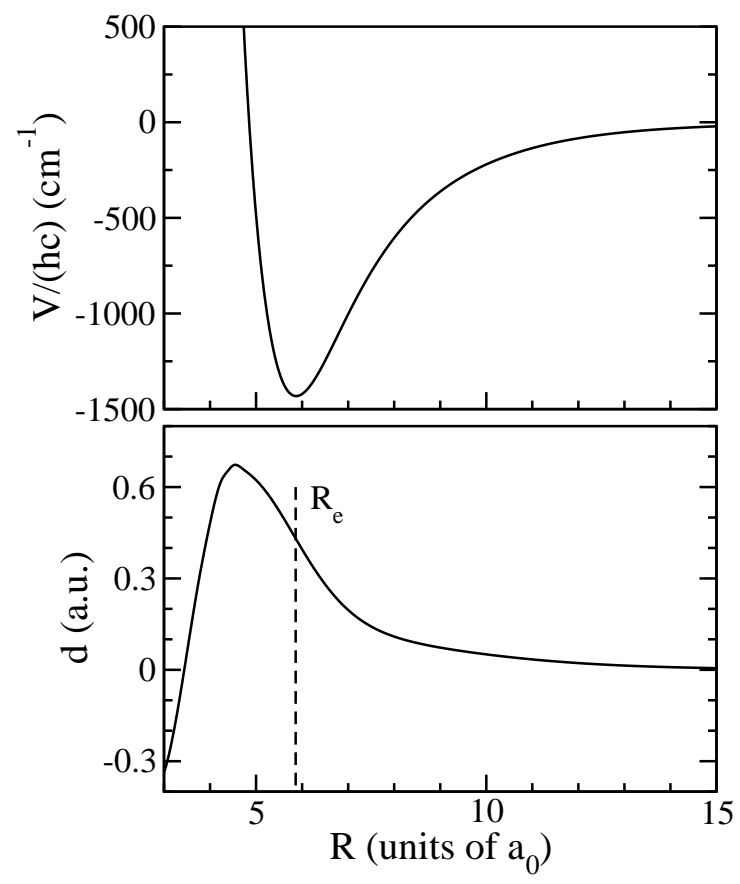

FIG. 2: Top panel: electronic $\mathrm{X}^{2} \Sigma^{+}$ground state potential of LiMg; bottom panel: permanent dipole moment, where a vertical dashed line indicates the equilibrium separation of the ground state potential; There the dipole moment is 0.44 a.u., while the vibrationally averaged value for the $v=0$ level is 0.413 a.u..

\section{LiCa molecule}

Currently, there is no reliable experimental data for the ground state potential of the LiCa molecule in the literature. However, preliminary experimental results on the electronic properties of the ground-state LiCa potential based on high resolution spectroscopy were presented at the EGAS conference in 2010 [11. They gave the molecular constants $D_{e} /(h c)=2607.8(100) \mathrm{cm}^{-1}, R_{e}=$ $6.3415(5) a_{0}$, and $\omega_{e} /(h c)=195.2 \mathrm{~cm}^{-1}$ for ${ }^{7} \mathrm{Li}^{40} \mathrm{Ca}$. Earlier theoretical predictions for the $\mathrm{LiCa}$ ground state give a dissociation energy of $D_{e} /(h c)=2177.7 \mathrm{~cm}^{-1}$ and $R_{e}=6.65 a_{0}$ using density functional theory [29]. Later a configuration-interaction calculation involving an effective pseudo-potential 38 predicted $D_{e} /(h c)=2355$ $\mathrm{cm}^{-1}$ and $R_{e}=6.23 a_{0}$. An all-electron $a b$ initio study of LiCa in Ref. 39 reported $D_{e} /(h c)=1935.7 \mathrm{~cm}^{-1}$ and $R_{e}=6.44 a_{0}$. Experimental two-photon ionization spectroscopy reported in the same work found that $R_{e}$ equals to $6.3865 a_{0}$. The substantial discrepancy in the reported characteristics and lack of the experimental data on the electronic structure of this molecule has initiated our study.

An accurate treatment of the $\mathrm{LiCa}$ molecule with presumably weak bonding requires a massive inclusion of correlation effects. To satisfy this requirement we apply two coupled-cluster approaches, $\operatorname{UCCSD}(\mathrm{T})$ and UCCSDT, that allow us to introduce higherorder correlation effects. To probe the influence of basis sets on the LiCa characteristics we used two different basis sets for each atom. These are allelectron basis sets with quadruple-zeta quality. Basis sets (def2-QZVPP) for Li:(15s6p2d1f)/[6s4p2d1f] and Ca:(24s18p6d3f)/[11s6p4d3f] functions are taken from Ref. 41. Basis sets (aug-cc-pCVQZ) with Li:(15s9p5d3f1g)/[8s7p5d3f1g] 34 and Ca:(25s19p10d4f2g)/[10s9p7d4f2g] [37] functions include larger correlation expansions.

We find that the molecular characteristics depend not only basis set but also on the number of correlated electrons $n_{e \ell}$. For example, for a 3-electron correlation one $2 s^{1}$ valence electron of $\mathrm{Li}$ and two $4 s^{2}$ valence electrons of $\mathrm{Ca}$ are correlated, whereas for 13-electron correlation all electrons of $\mathrm{Li}$ and ten $3 s^{2} 3 p^{6} 4 s^{2}$ electrons of $\mathrm{Ca}$ are included in the active space. We conclude that using the UCCSD(T) method and the more advanced basis set (aug-cc-pCVQZ) leads to an increase of $D_{e} /(h c)$ and $d_{e}$ by $\approx 5 \%$. For the UCCSDT method we expect similar trends. We believe that the UCCSDT calculation with the basis set (aug-cc-pCVQZ) provides the most accurate electronic potential and dipole moment. These are shown in Fig. 3. Moreover, this calculation is in a good agreement with the experimental molecular constants reported by Ref. [11.

The upper panel of Fig. 3 shows the potential energy curve of the $\mathrm{X}^{2} \Sigma^{+}$state of LiCa as a function of interatomic separation. The permanent dipole moment of this state as a function of $R$ is presented in the lower panel. A complete list of the $N=0$ vibrational energies of the ground state is presented in Table III]

TABLE III: All vibrational energies of the ${ }^{7} \mathrm{Li}^{40} \mathrm{Ca} \mathrm{X}^{2} \Sigma^{+}$ ground state potential for the rotational state $N=0$. The potential is based on the $\mathrm{UCCSD}(\mathrm{T}) / \mathrm{aug}-\mathrm{cc}-\mathrm{PCVQZ}$ calculation.

\begin{tabular}{rccc}
\hline \hline$\nu$ & Energy $/(h c)\left(\mathrm{cm}^{-1}\right)$ & $\nu$ & Energy/(hc) $\left(\mathrm{cm}^{-1}\right)$ \\
0 & -2428.02 & 14 & -442.18 \\
1 & -2232.09 & 15 & -366.32 \\
2 & -2042.75 & 16 & -298.77 \\
3 & -1861.87 & 17 & -239.22 \\
4 & -1688.77 & 18 & -187.31 \\
5 & -1524.31 & 19 & -142.70 \\
6 & -1368.30 & 20 & -105.03 \\
7 & -1221.05 & 21 & -73.95 \\
8 & -1082.74 & 22 & -49.08 \\
9 & -953.39 & 23 & -30.02 \\
10 & -833.10 & 24 & -16.33 \\
11 & -721.92 & 25 & -7.41 \\
12 & -619.79 & 26 & -2.47 \\
13 & -526.60 & 27 & -0.42 \\
\hline \hline
\end{tabular}




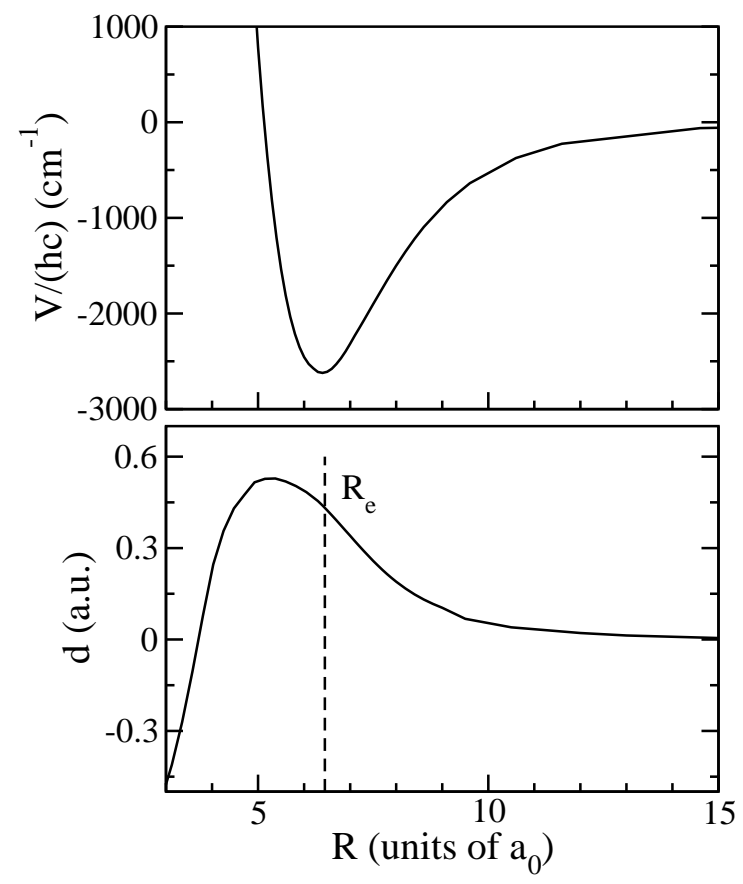

FIG. 3: Top panel: electronic ground state ${ }^{2} \Sigma_{1 / 2}^{+}$potential of LiCa; bottom panel: permanent dipole moment of the ground state of LiCa. The vertical dashed line indicates the equilibrium separation of the ground state potential. At this separation the dipole moment is 0.044 a.u. The vibrationally averaged dipole moment with the $v=0$ wave function is 0.437 a.u.

\section{LiSr molecule}

To our knowledge there exist no data on the LiSr ground-state potential based on experimental observation. We are only able to compare our results to the theoretical results of Ref. [6]. The calculation of Ref. 6] uses an effective core and core polarization potentials that are incorporated in a configuration-interaction approach. Their molecular constants are $R_{e}=6.57 a_{0}, D_{e} /(h c)=$ $2587 \mathrm{~cm}^{-1}, \omega_{e} /(h c)=184.9 \mathrm{~cm}^{-1}, B_{e} /(h c)=0.21 \mathrm{~cm}^{-1}$, and $d_{e}=0.13$ a.u. In the present study we use different computational methods to predict these characteristics.

We perform calculations for $\mathrm{LiSr}$ that are similar to those for LiCa. We use UCCSD(T) and UCCSDT methods with three different basis sets. The inner electrons of the closed $1 s^{2} 2 s^{2} \ldots 3 d^{10}$ shells of Sr are chemically inactive and excluded from the correlation calculation. The $1 s^{2} 2 s^{1}$ electrons of $\mathrm{Li}$ and the ten $5 s^{2} 4 s^{2} 4 p^{6}$ electrons of $\mathrm{Sr}$, are included in the all-order correlation calculation.

In the first calculation we use the basis set def2-QZVPP with Li:(15s6p2d1f)/[6s4p2d1f] and Sr:(8s8p5d3f)/[7s5p4d3f] functions [41] and the Stuttgart ECP28MDF relativistic effective core potential (ECP) 40. In a second calculation we use the basis set "[42]" with Li: (14s8p4d2f)/[7s6p4d2f]

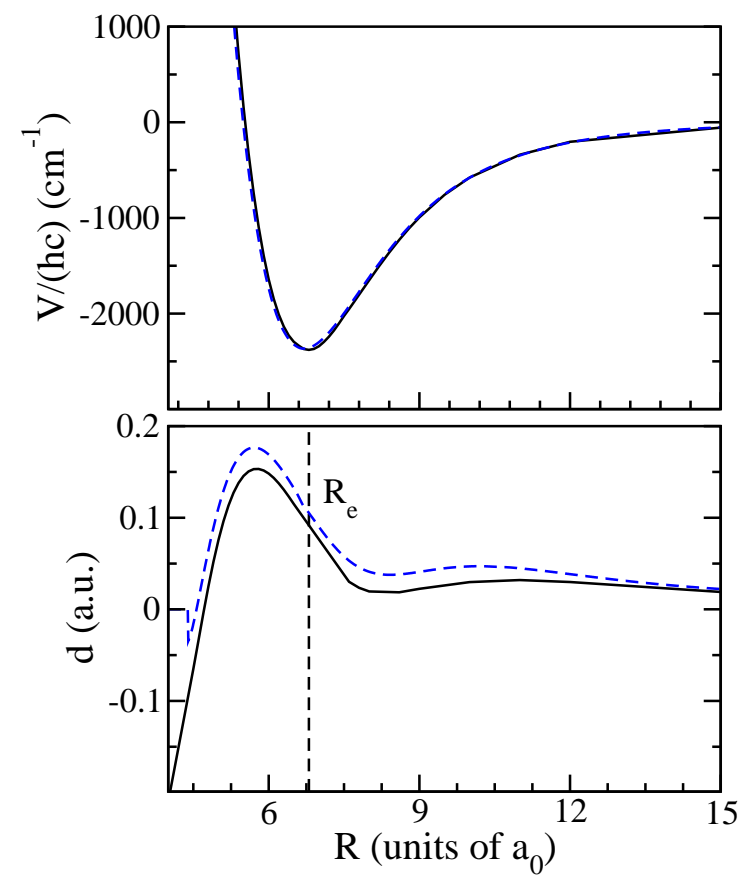

FIG. 4: Top panel: electronic ground-state ${ }^{2} \Sigma+$ potential of LiSr; bottom panel: permanent dipole moment of the ground state of LiSr. The potentials and dipole moments are obtained by two different methods: UCCSDT (solid curve) and $\operatorname{UCCSD}(\mathrm{T})$ (dashed curve). The vertical dashed line in the bottom panel indicates the equilibrium separation of the ground state potential. The vibrationally averaged dipole moment with the $v=0$ wave function is 0.111 a.u.;

and Sr: (14s11p5d4f1g)/[ 8s8p5d4f1g] functions. The effective core potential is from Ref. [42. Finally, we performed a calculation with a more-advanced all-electron basis set (aug-cc-pCV5Z) described by $\mathrm{Li}: \quad(18 \mathrm{~s} 12 \mathrm{p} 7 \mathrm{~d} 5 \mathrm{f} 3 \mathrm{~g} 1 \mathrm{~h}) /[10 \mathrm{~s} 9 \mathrm{p} 7 \mathrm{~d} 5 \mathrm{f} 3 \mathrm{~g} 1 \mathrm{~h}]$ and $\mathrm{Sr}$ : (23s19p12d4f2g)/[11s10p7d4f2g] functions from [35]. The UCCSDT calculation is time consuming and has been performed for basis set "[42]" only.

Results of the most advanced calculation by the UCCSDT method with the basis set "[42]" and the $\mathrm{UCCSD}(\mathrm{T})$ method with basis set (aug-cc-pCV5Z) are shown in Fig. 4. We see that the potential energy of the two calculations nearly coincide, whereas the dipole moment curves in the bottom panel are slightly shifted relative to each other. We believe that this difference is within the accuracy of our calculation. The largest discrepancy of about $7 \%$ with the results of Ref. [6] occurs at large internuclear separation, whereas at $R_{e}$ the dipole moment $d_{e}$ differs by $2 \%$ to $3 \%$ only.

The DVR calculation of the bound state energy gives $D_{0} /(h c)=2275.59 \mathrm{~cm}^{-1}$ and $\omega /(h c)=182.2 \mathrm{~cm}^{-1}$. Table IV lists all $N=0$ bound-state energies for ${ }^{7} \mathrm{Li}^{88} \mathrm{Sr}$. 
TABLE IV: All vibrational energies of the ${ }^{7} \mathrm{Li}^{88} \mathrm{Sr} \mathrm{X}^{2} \Sigma^{+}$ ground state potential for the rotational state $N=0$. The potential is based on the $\operatorname{UCCSD}(\mathrm{T})$ calculation with basis set 35

\begin{tabular}{rccc}
\hline \hline$\nu$ & Energy/(hc) $\left(\mathrm{cm}^{-1}\right)$ & $\nu$ & Energy $/(h c)\left(\mathrm{cm}^{-1}\right)$ \\
0 & -2275.59 & 15 & -397.78 \\
1 & -2099.56 & 16 & -331.60 \\
2 & -1931.06 & 17 & -272.33 \\
3 & -1769.35 & 18 & -219.76 \\
4 & -1614.41 & 19 & -173.66 \\
5 & -1466.66 & 20 & -133.76 \\
6 & -1326.14 & 21 & -99.83 \\
7 & -1192.91 & 22 & -71.56 \\
8 & -1067.12 & 23 & -48.65 \\
9 & -948.89 & 24 & -30.80 \\
10 & -838.24 & 25 & -17.64 \\
11 & -735.17 & 26 & -8.73 \\
12 & -639.65 & 27 & -3.42 \\
13 & -551.65 & 28 & -0.86 \\
14 & -471.08 & 29 & -0.04 \\
\hline \hline
\end{tabular}

\section{E. LiYb molecule}

Recently, two extensive quantum-chemical calculations of the ground and excited states of the $\mathrm{LiYb}$ molecule have been reported by Zhang et al. 8, and by Gopakumar et al. 9]. The first calculation used both MRCI and UCCSD(T) methods. Scalar relativistic effects were included by the Douglas-Kroll Hamiltonian and an effective core potential. Their molecular constants for the $\mathrm{X}^{2} \Sigma^{+}$ ground state of ${ }^{7} \mathrm{Li}^{172} \mathrm{Yb}$ are the following: $R_{e}=6.681 a_{0}$, $D_{e} /(h c)=1577 \mathrm{~cm}^{-1}, \omega_{e} /(h c)=147.36 \mathrm{~cm}^{-1}$. Different treatments of the $\mathrm{Yb}$ core electrons lead to different values of the dipole moment. A RECP-pseudo-potential calculation gave $d_{e}=0.022$ a.u., while an all-electron DKH3 calculation gave a smaller $d_{e}$ of 0.011 a.u. Results of a calculation by [9] were based on both the CASPT2 perturbation theory and the $\operatorname{CCSD}(\mathrm{T})$ method. Relativistic effects were taken into account through the DouglasKroll-Hess Hamiltonian. Their best values of molecular constants are: $R_{e}=6.669 a_{0}, D_{e} /(h c)=1421.96 \mathrm{~cm}^{-1}$, $\omega_{e} /(h c)=135.54 \mathrm{~cm}^{-1}$.

Our goal here is to perform an independent calculation of the ground state potential and permanent dipole moment of LiYb using the UCCSD(T) method with another effective ECP28MWB pseudo-potential and with basis sets for Li: (15s6p2d1f)/[6s4p2d1f] and Yb: (14s13p10d8f6g)/[10s8p5d4f3g] functions from [44].

Results of our calculation are shown in Fig. 5. The upper panel of Fig. 5 shows the ground state potential of LiYb, which is significantly deeper than the potentials obtained in Refs. [8, 9]. The permanent dipole moment is presented in the bottom panel. It is small and agrees

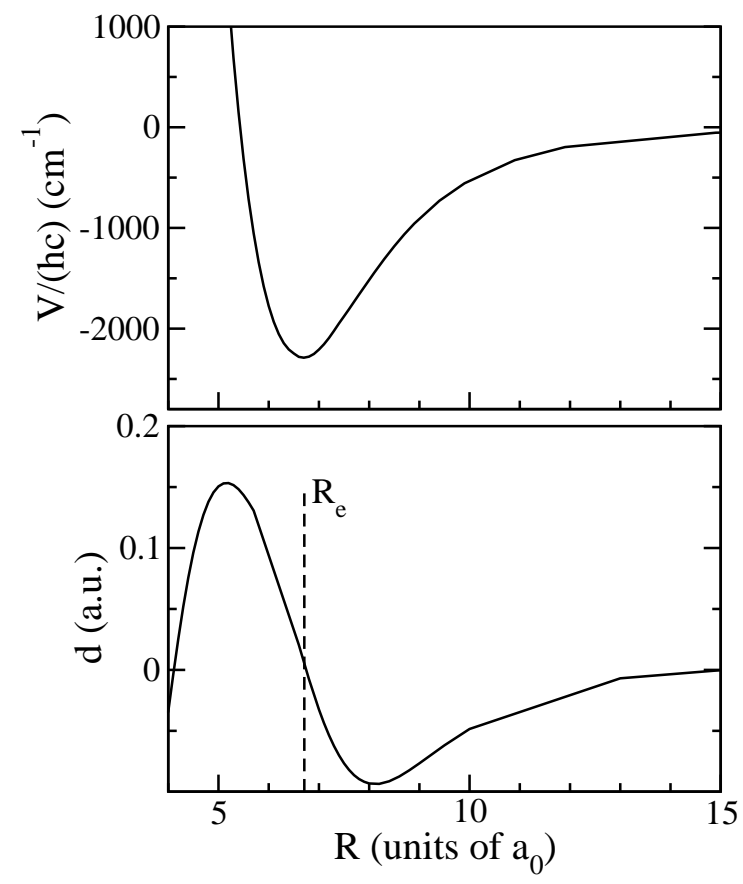

FIG. 5: Top panel: electronic ground-state ${ }^{2} \Sigma^{+}$potential of $\mathrm{LiYb}$; bottom panel: permanent dipole moment of the ground state. The vertical dashed line in the bottom panel indicates the equilibrium separation of the ground state potential;

well with the DKH3 value of Ref. [8].

Table $\mathrm{V}$ shows all $N=0$ bound-state energies for ${ }^{7} \mathrm{Li}^{172} \mathrm{Yb}$.

\section{SUMMARY}

The objective of our research has been a systematic investigation of the $\mathrm{X}^{2} \Sigma^{+}$ground state properties of Ligroup II molecules. To achieve this goal we applied powerful quantum chemistry $\mathrm{UCCSD}(\mathrm{T})$ and UCCSDT methods with large basis sets to calculate potential energies and permanent dipole moments. Table VI displays molecular constants obtained in our calculations.

Figures 6 and 7 and Table VI] summarize our results. Figure 6 reveals a most striking irregularity in the dissociation energy and $R_{e}$ among the Li-group II molecules. The deepest and most shallow potentials belong to the $\mathrm{LiCa}$ and $\mathrm{LiMg}$ molecules, respectively.

Figure 7] shows dipole moment functions for all molecules considered in our study. There is some consistency in the maximum value of $d$ with the reduced mass of the molecule: heavier molecules have smaller dipole moment. The dipole moment averaged over the ground state vibrational wave function is always a few tenths of atomic units smaller than $d_{e}$. The LiSr and LiYb molecule have the smallest dipole moments among all molecules in this study. 


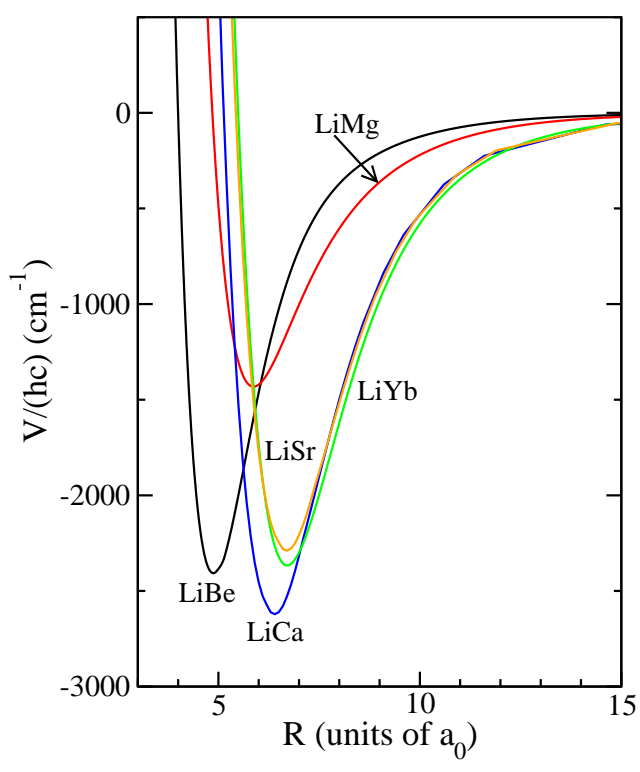

FIG. 6: Electronic ground state ${ }^{2} \Sigma^{+}$potentials of Li-group II molecules and $\mathrm{LiYb}$ as a function of interatomic separation.

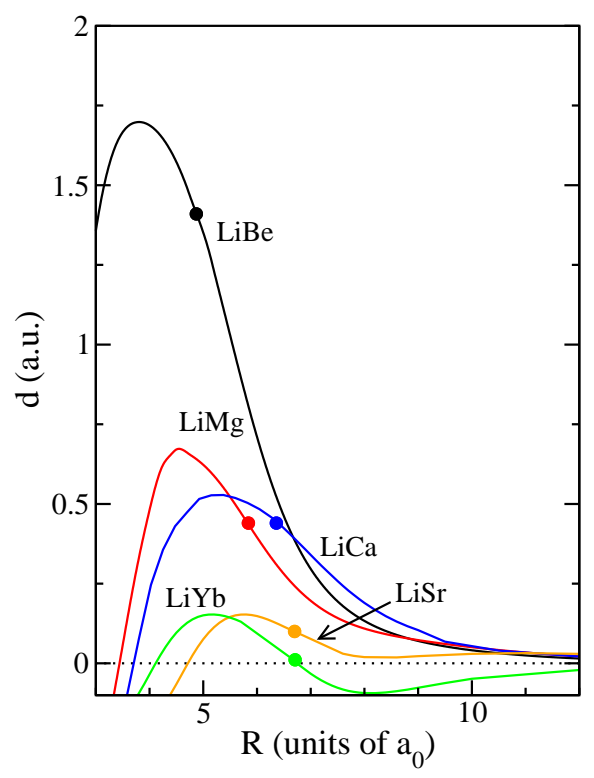

FIG. 7: Permanent dipole moment of the ground state of the Li-group II molecules and $\mathrm{LiYb}$ as a function of interatomic separation. Filled circes indicate dipole moment values at equiliblium separations of corresponding molecules.
TABLE V: All vibrational energies of the ${ }^{7} \mathrm{Li}^{172} \mathrm{Yb} \mathrm{X}^{2} \Sigma^{+}$ ground state potential for the rotational state $N=0$. The potential is based on the $\operatorname{UCCSD}(\mathrm{T})$ calculation with basis set 44

\begin{tabular}{rccc}
\hline \hline$\nu$ & Energy $/(h c)\left(\mathrm{cm}^{-1}\right)$ & $\nu$ & Energy/(hc) $\left(\mathrm{cm}^{-1}\right)$ \\
0 & -2277.12 & 16 & -349.63 \\
1 & -2103.98 & 17 & -289.41 \\
2 & -1938.15 & 18 & -235.73 \\
3 & -1778.86 & 19 & -188.38 \\
4 & -1626.11 & 20 & -147.10 \\
5 & -1480.30 & 21 & -111.67 \\
6 & -1341.45 & 22 & -81.82 \\
7 & -1209.63 & 23 & -57.26 \\
8 & -1084.99 & 24 & -37.70 \\
9 & -967.64 & 25 & -22.84 \\
10 & -857.62 & 26 & -12.36 \\
11 & -754.90 & 27 & -5.66 \\
12 & -659.49 & 28 & -1.94 \\
13 & -571.36 & 29 & -0.36 \\
14 & -490.44 & 30 & -0.005 \\
15 & -416.57 & & \\
\hline \hline
\end{tabular}

Table VI presents molecular constants calculated by the RCCSD $(\mathrm{T})$, UCCSD $(\mathrm{T})$ and UCCSDT methods using several large basis sets available in the literature. Comparison of our most accurate (either $\operatorname{UCCSD}(\mathrm{T})$ or UCCSDT) calculations with existing experimental measurements of the molecular constants shows that we are able to reproduce the bond length $\left(R_{e}\right)$, harmonic frequency $\left(\omega_{e}\right)$, and dissociation energy $\left(D_{e}\right)$ within $5 \%$, $8 \%$, and $6 \%$, respectively.

The dipole moment as a function $R$ is calculated in terms of a charge transfer and induced charge transfer. These two contributions have opposite signs over a large range of internuclear separations. We conclude from our calculations that charge transfer from the Li-atom to the alkaline-earth atom prevails at all separations. This trend is reversed for LiYb at $R>7 a_{0}$.

We also highlight the importance of the correlation effects in calculations of the alkali-alkaline-earth molecules. We have gathered numerical evidence to show that molecular constants of these molecules, such as dissociation energy and permanent dipole moment of the ground state, are extremely sensitive to high order correlations. We believe that the best values of these characteristics are obtained by using the UCCSDT method, where correlation effects are taken into account at all orders.

Finally, we want to emphasize that $\mathrm{LiBe}$ and $\mathrm{LiMg}$ are not likely candidates for ultracold studies because of the difficulty of cooling Be or Mg. However, LiCa looks quite promising, since a $\mathrm{Ca} \mathrm{BEC}[45$, has been made, and its dipole moment is comparable to the known RbCs case [46. The ${ }^{2} \Sigma^{+}$ground state gives an extra spin degree of freedom relative to the ${ }^{1} \Sigma^{+}$RbCs species, thus 
TABLE VI: Molecular constants for the $\mathrm{X}^{2} \Sigma^{+}$state of LiBe, LiMg, LiCa, LiSr, and LiYb obtained by the RCCSD $(\mathrm{T})$, $\mathrm{UCCSD}(\mathrm{T})$ and UCCSDT methods. Here, $1 a_{0}=0.0529177$ $\mathrm{nm}$ is the Bohr radius and a dipole moment of 1 a.u. corresponds to $1 e a_{0}$, where $e$ is the electron charge. Also $h$ is Planck's constant and $c$ the speed of light.

\begin{tabular}{|c|c|c|c|c|}
\hline Description & $\left.\mid \begin{array}{l}R_{e} \\
\left(a_{0}\right)\end{array}\right)$ & $\begin{array}{l}\omega_{e} /(h c) \\
\left(\mathrm{cm}^{-1}\right)\end{array}$ & $\begin{array}{l}D_{e} /(h c) \\
\left(\mathrm{cm}^{-1}\right)\end{array}$ & $\begin{array}{l}d_{e} \\
\text { (a.u.) }\end{array}$ \\
\hline \multicolumn{5}{|c|}{${ }^{7} \mathbf{L i}^{9} \mathrm{Be}$} \\
\hline \multicolumn{5}{|l|}{$\overline{\mathrm{UCCSD}(\mathrm{T})}$} \\
\hline aug-cc-pV5Z-DK & 4.873 & 299.5 & 2406 & 1.41 \\
\hline Experiment 32 & 4.894 & & & \\
\hline \multicolumn{5}{|c|}{${ }^{7} \mathbf{L i}^{24} \mathbf{M g}$} \\
\hline $\operatorname{RCCSD}(\mathrm{T})$ & & & & \\
\hline $\begin{array}{r}\text { aug-cc-pCVQZ } \\
\mathbf{U C C S D}(\mathbf{T})\end{array}$ & 5.86 & 174.4 & 1417 & 0.32 \\
\hline aug-cc-pV5Z-DK & 5.87 & 206.1 & 1432 & 0.44 \\
\hline Experiment 23] & 5.9 & 190 & $1423^{a}$ & \\
\hline \multicolumn{5}{|c|}{${ }^{7} \mathbf{L i}^{40} \mathbf{C a}$} \\
\hline \multicolumn{5}{|l|}{$\operatorname{UCCSD}(\mathrm{T})$} \\
\hline def2-QZVPP & 6.410 & 196.0 & 2320 & 0.438 \\
\hline aug-cc-pCVQZ & 6.358 & 205.5 & 2460 & 0.456 \\
\hline \multicolumn{5}{|l|}{ UCCSDT } \\
\hline aug-cc-pCVQZ & 6.357 & 207.1 & 2607 & 0.440 \\
\hline Experiment [1] & $6.3415(5)$ & 195.2 & $2607.8(100)$ & \\
\hline \multicolumn{5}{|c|}{${ }^{7} \mathbf{L i}^{88} \mathbf{S r}$} \\
\hline \multicolumn{5}{|l|}{$\operatorname{UCCSD}(\mathrm{T})$} \\
\hline def2-QZVPP & 6.766 & 167.0 & 2165 & 0.109 \\
\hline basis of 42 & 6.712 & 183.0 & 2302 & 0.117 \\
\hline aug-cc-pCV5Z & 6.700 & 182.2 & 2367 & 0.112 \\
\hline \multicolumn{5}{|l|}{ UCCSDT } \\
\hline basis of 42 ] & 6.711 & 184.2 & 2401 & 0.096 \\
\hline \multicolumn{5}{|c|}{${ }^{7} \mathbf{L i}^{172} \mathbf{Y b}$} \\
\hline \multicolumn{5}{|l|}{$\mathrm{UCCSD}(\mathrm{T})$} \\
\hline basis of 44 & 6.710 & 181.5 & 2289 & 0.011 \\
\hline
\end{tabular}

${ }^{a}$ This value is estimated from $D_{0}$ of Ref. 23 more quantum control possibilities. While ultracold $\mathrm{Li}$ $+\mathrm{Sr}$ and $\mathrm{Li}+\mathrm{Yb}$ mixtures are possible for making the molecules, the dipole moments of the ground state molecules are rather modest. It remains to be determined how best to make the molecules from the trapped atoms. One possibility is to make "preformed pairs" in a dual species optical lattice [47, 48, and then use intercombination line photoassociation followed by STIRAP 49. Although all of these molecular species are expected to be highly chemical reactive in their ground states since the formation of the dimers is exotermic LiCa might have a large enough dipole moment to allow it to be protected against destructive collisions by aligning it with an electric field in a 1D optical lattice [1, 50, 51].

\section{ACKNOWLEDGMENTS}

The Temple University team and PSJ acknowledge support by an AFOSR MURI grant on ultracold polar molecules. Work at Temple University was also supported by NSF Grant PHY-1005453, and J. K. would like to thank for the financial support through NSF Grant CHE-0848110 to Millard H. Alexander.
[1] A. Micheli, G. K. Brennen, and P. Zoller, Nature Phys. 2, 341 (2006).

[2] L. K. Sørensen, S. Knecht, T. Fleig, and C. M. Marian, J. Phys. Chem. 113, 12607 (2009).

[3] L. D. Carr, D. DeMille, R. Krems, and J. Ye, New J. Phys. 11, 055049 (2009).

[4] J. Perez-Rios, F. Herrera, and R. V. Krems, New J. Phys. 12, 103007 (2010).

[5] P. S. Żuchowski, J. Aldegunde, and J. M. Hutson, Phys. Rev. Lett. 105, 153201 (2010).

[6] R. Guérout, M. Aymar, and O. Dulieu, Phys. Rev. A 82, 042508 (2010).
[7] M. Okano, H. Hara, M. Muramatsu, K. Doi, S. Uetake, Y. Takasu, and Y. Takahashi, Appl. Phys. B: Lasers Opt. 98, 691 (2010).

[8] P. Zhang, H. R. Sadeghpour, A. Dalgarno, J. Chem. Phys. 133, 044306 (2010).

[9] G. Gopakumar, M. Abe, B. P. Das, M. Hada, and K. Hirao, J. Chem. Phys. 133, 124317 (2010).

[10] V. V. Ivanov et al., Phys. Rev. Lett. 106, 153201 (2011).

[11] M. Ivanova, A. Pashov, A. Stein, H. Knöckel, and E. Tiemann, (private communication) (2011).

[12] T.V. Tscherbul and R.V. Krems, Phys. Rev. Lett. 97, 083201 (2006).

[13] J. J. Hudson, B. E. Sauer, M. R. Tarbutt, and E. A. 
Hinds, Phys. Rev. Lett. 89, 023003 (2002).

[14] P. O. Fedichev, Yu. Kagan, G. V. Shlyapnikov, and J. T. M. Walraven, Phys. Rev. Lett. 77, 2913 (1996).

[15] J. L. Bohn and P. S. Julienne, Phys. Rev. A 54, R4637 (1996).

[16] F. K. Fatemi, K. M. Jones, and P. D. Lett, Phys. Rev. Lett. 85, 4462 (2000).

[17] M. Theis, G. Thalhammer, K. Winkler, M. Hellwig, G. Ruff, R. Grimm, and J. H. Denschlag, Phys. Rev. Lett. 93, 123001 (2004).

[18] R. Ciurylo, E. Tiesinga, and P. S. Julienne, Phys. Rev. A 71, 030701 (2005).

[19] G. Thalhammer, M. Theis, K. Winkler, R. Grimm, J. H. Denschlag, Phys. Rev. A 71, 033404 (2005).

[20] K. M. Jones, E. Tiesinga, P. D. Lett, and P. S. Julienne, Rev. Mod. Phys. 78, 483 (2006).

[21] K. Enomoto, K. Kasa, M. Kitagawa, and Y. Takahashi, Phys. Rev. Lett. 101, 203201 (2008).

[22] G. Pichler, A. M. Lyyra, P. D. Kleiber, W. C. Stwalley, R. Hammer, and K. M. Sando, Chem. Phys. Lett. 156, 467 (1989).

[23] K. R. Berry and M. A. Duncan, Chem. Phys. Lett. 279, 44 (1997).

[24] G. Haeffler, D. Hanstorp, I. Kiyan, A. E. Klinkmueller, U. Ljungblad, D. J. Pegg, Phys. Rev. A 53, 4127 (1996).

[25] C. W. Bauschlicher, Jr. S. R. Langhoff, and H. Partridge, J. Chem. Ph ys. 96, 1240 (1992).

[26] J.F. Stanton et al., J. Chem. Theor. Comp. 4, 64 (2008).

[27] H.-J. Werner et al., MOLPRO, Version 2008.1, a package of ab initio programs.

[28] T. J. Lee et al. Theor. Chem. Acta 75, 81 (1989).

[29] R. O. Jones J. Chem. Phys. 72, 3197 (1980).

[30] I. Fischer, V. E. Bondybey, P. Rosmus, and H.-J. Wener, Chem Phys. 151, 295 (1991).

[31] M. M. Marino and W. C. Ermler, J. Chem. Phys. 96, 3756 (1992).

[32] R. Schlachta, I. Fischer, P. Rosmus, and V. E. Bondybey, Chem. Phys. Lett. 170, 485 (1990).

[33] S. F. Boys and F. Bernardi, Mol. Phys. 19, 553 (1970).

[34] T.H. Dunning, Jr. J. Chem. Phys. 90, 1007 (1989).

[35] B. Prascher, D.E. Woon, K.A. Peterson, T.H. Dun- ning, Jr., and A.K. Wilson, Theor. Chem. Acc.,128,69-82 (2011)

[36] D.T.Colbert and W.H.Miller, J. Chem. Phys. 96, 1982 (1992).

[37] J. Koput and K.A. Peterson, J. Phys. Chem. A 106, 9595 (2002).

[38] A. R. Allouche and M. Aubert-Frecon, Chem. Phys. Lett. 222, 524 (1994).

[39] L. M. Russon, G. K. Rothschopf, M. D. Morse, A. I. Boldyrev, and J. Simons, J. Chem. Phys. 109, 6655 (1998).

[40] I. Lim, P. Schwerdtfeger, B. Metz, and H. Stoll, J. Chem. Phys. 122, 104103 (2005).

[41] F. Weigend and R. Ahlrichs, Phys. Chem. Chem. Phys. 7, 3297 (2005).

[42] I.S. Lim, H. Stoll, P. Schwerdtfeger, J. Chem. Phys. 124, 034107 (2006).

[43] M. Dolg, H. Stoll, H. Preuss, J. Chem. Phys. 90, 1730 (1989).

[44] X. Cao, M. Dolg, J. Molec. Struct. Theochem. 581 139, (2002).

[45] S. Kraft et al., Phys. Rev. Lett. 103, 130401 (2009).

[46] M. Aymar and O. Dulieu, J. Chem. Phys. 122, 204302 (2005).

[47] B. Damski, L. Santos, E. Tiemann, M. Lewenstein, S. Kotochigova, P. Julienne, and P. Zoller, Phys. Rev. Lett. 90, 110401 (2003).

[48] J. K. Freericks, M. M. Maka, Anzi Hu, Thomas M. Hanna, C. J. Williams, and P. S. Julienne, and R. Lemaski, Phys. Rev. A 81, 011605(R) (2010).

[49] K.-K. Ni, S. Ospelkaus, M. H. G. de Miranda, A. Peer, B. Neyenhuis, J. J. Zirbel, S. Kotochigova, P. S. Julienne, D. S. Jin, J. Ye, Science 322, 231 (2008).

[50] M. H. G. de Miranda, A. Chotia, B. Neyenhuis, D.Wang, G. Qumner, S. Ospelkaus, J. L. Bohn, J. Ye, and D. S. Jin, Nature Phys. 7, 502 (2011).

[51] P. S. Julienne, T. M. Hanna, and Z. Idziaszek, Phys. Chem. Chem. Phys. Advanced Article DOI:10.1039/C1CP21270B. 